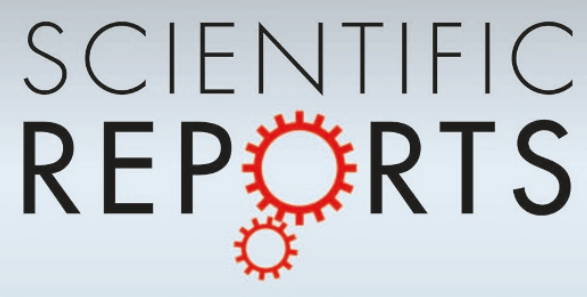

OPEN

SUBJECT AREAS:

QUANTUM OPTICS

QUANTUM INFORMATION

Received

24 July 2013

Accepted

20 March 2014

Published

11 April 2014

Correspondence and requests for materials should be addressed to

F.D. (fgdeng@bnu. edu.cn)

* These authors contributed equally to this work.

\section{Hyper-parallel photonic quantum computation with coupled quantum dots}

\author{
Bao-Cang Ren* \& Fu-Guo Deng*
}

Department of Physics, Applied Optics Beijing Area Major Laboratory, Beijing Normal University, Beijing 100875, China.

It is well known that a parallel quantum computer is more powerful than a classical one. So far, there are some important works about the construction of universal quantum logic gates, the key elements in quantum computation. However, they are focused on operating on one degree of freedom (DOF) of quantum systems. Here, we investigate the possibility of achieving scalable hyper-parallel quantum computation based on two DOFs of photon systems. We construct a deterministic hyper-controlled-not (hyper-CNOT) gate operating on both the spatial-mode and the polarization DOFs of a two-photon system simultaneously, by exploiting the giant optical circular birefringence induced by quantum-dot spins in double-sided optical microcavities as a result of cavity quantum electrodynamics (QED). This hyper-CNOT gate is implemented by manipulating the four qubits in the two DOFs of a two-photon system without auxiliary spatial modes or polarization modes. It reduces the operation time and the resources consumed in quantum information processing, and it is more robust against the photonic dissipation noise, compared with the integration of several cascaded CNOT gates in one DOF.

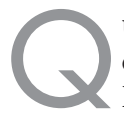
uantum information processing, based on quantum mechanics theory, has largely improved the methods of dealing and transmitting information in quantum computation ${ }^{1}$ and quantum communication ${ }^{2}$. Parallel quantum computation provides a novel way to precisely control and manipulate the states of quantum systems, which is much faster than the conventional computation in principle. Quantum logic gate is a key element in quantum computation. It is very important to find an effective physical realization of quantum logic gates. Many proposals have been proposed to implement controlled-not (CNOT) gates or controlled phaseflip (CPF) gates both in theory and in experiment by using various quantum systems, such as ion trap ${ }^{3}$, free electron $^{4}$, cavity-QED system ${ }^{5,6}$, nuclear magnetic resonance ${ }^{7-9}$, quantum $\operatorname{dot}^{10}$, superconducting charge qubits ${ }^{11}$, and polarization states of single photons ${ }^{12-16}$. In 2001, Knill, Laflamme, and Milburn ${ }^{12}$ showed that a CNOT gate on photonic qubits with the maximal success probability of $3 / 4$ could be constructed, resorting to only singlephoton sources, single-photon detectors, and linear optical elements. In contrast, a deterministic photonic CNOT gate can be constructed with nonlinear optics. In 2004, Nemoto and Munro ${ }^{14}$ constructed a nearly deterministic CNOT gate with weak cross-Kerr nonlinearity, ancilla photons, and feed-forward operations. The key element of this proposal is the quantum nondemolition detector using the nonlinear optics of weak cross-Kerr media. At present, a giant Kerr nonlinearity is still a challenge, even with electromagnetically induced transparency ${ }^{17}$, because the initial phase shift achieved at the single-photon level ${ }^{18}$ is only in the order of $10^{-5}$. Moreover, it is not clear whether these nonlinearities are sufficient for the natural implementation of single-photon qubit gates or not $^{19}$. Cavity QED is a promising candidate for obtaining large nonlinear phase shifts with the dipole emitter resonant with the cavity mode ${ }^{20}$. In 2004, Duan and Kimble ${ }^{15}$ proposed a scheme to implement a CPF gate for photon systems with the nonlinear phase shifts caused by a single atom trapped in an optical cavity. In 2010, Koshino et al. ${ }^{16}$ presented a method to implement a deterministic photon-photon $\sqrt{S W A P}$ gate using a threelevel system embedded in an optical cavity. The nonlinear approaches using multimode optical parametric oscillator $^{21}$ and coherent photon conversion ${ }^{22}$ can also be used to implement deterministic quantum computing processes.

In order to implement quantum computer, there are some requirements for physical systems: efficient manipulation, reading out the state of an individual qubit, strong coupling between qubits, weak coupling to environment, and scalability. Recently, a solid-state quantum system based on an electron spin in a quantum dot (QD) inside a microcavity (QD-cavity) has attracted much attention with its good optical property and scalability. In 2008, Hu et al. ${ }^{23,24}$ proposed a quantum nondemolition method using the giant optical circular birefringence of a one-side QD-cavity system, and they pointed out that the interaction of circularly polarized lights and 
double-sided QD-cavity system can be used to construct entanglement beam splitter $(\mathrm{EBS})^{25}$ in 2009 . With the optical property of QDcavity systems, many quantum information processes can be implemented, such as entanglement generation and Bell-state analysis ${ }^{23-26}$, quantum gates ${ }^{27-31}$, hyper-entangled Bell-state analysis ${ }^{32,33}$, and quantum repeater ${ }^{34}$. In 2010, Bonato et al. ${ }^{27}$ constructed a hybrid quantum CNOT gate on an electron-spin qubit and a photonic qubit using the EBS effect of a double-sided QD-cavity system.

It is well known that any universal quantum computation can be realized with two-qubit gates assisted by one-qubit rotations in quantum network theory ${ }^{35}$. In practice, there are many obstacles required to be overcome both in theory and in technology, such as the sheer number of element gates in quantum logic circuits, the implementation of element gates, and so on. So far, there are many important works on the construction of CNOT gates and CPF gates operating on one degree of freedom (DOF) of quantum systems (such as the polarization DOF of photon systems or the spin DOF of electron systems). Usually, the spatial-mode (polarization) DOF of photon systems is used to assist the construction of quantum logic gates that operate on the polarization (spatial-mode) DOF, in which the spatial-mode (polarization) DOF is consumed after the operation. In principle, both the spatial-mode and the polarization DOFs of photon systems can be used as the qubits for universal quantum gates on two DOFs of quantum systems. In this paper, we investigate the possibility of achieving scalable hyper-parallel quantum computation based on two DOFs of photon systems without using the auxiliary spatial modes or polarization modes, which can reduce the resources required for quantum logic circuits (or storage process) and depress the photonic dissipation in quantum information processing, compared with the integration of several cascaded CNOT gates in one DOF. We construct a deterministic hyper-controlled-not (hyper-CNOT) gate operating on both the spatial-mode and the polarization DOFs of a photon pair simultaneously, resorting to the giant optical circular birefringence induced by quantum-dot spins in double-sided optical microcavities as a result of cavity QED. This hyper-CNOT gate is useful for the quantum information protocols with multiqubit systems, and it decreases the interaction times between the photons required in quantum information processing.

\section{Results}

Quantum-dot-cavity system. Let us consider a singly charged QD [e.g. self-assembled $\operatorname{In}(\mathrm{Ga}) \mathrm{As}$ QD or GaAs interfacial QD] confined in a double-sided optical resonant microcavity. The QD is located at the center of a double-sided cavity, shown in Fig. 1(a). The two distributed Bragg reflectors are made partially reflective and low loss for on-resonance transmission, and the double-sided cavity is a two-mode one which supports both polarization modes ${ }^{36}$. With an excess electron injected into QD, the singly charged QD shows the optical resonance of a negatively charged exciton $X^{-}$that consists of two electrons bound to one hole ${ }^{37}$. According to Pauli's exclusion principle, $X^{-}$shows spin-dependent transitions interacting with circularly polarized lights ${ }^{38}$, shown in Fig. 1(b). The left-circularlypolarized light $|L\rangle\left(S_{z}=+1\right)$ couples the excess electron-spin state $|\uparrow\rangle$ to create $X^{-}$in the state $|\uparrow \downarrow \uparrow\rangle$ with two electron spins antiparallel, while the right-circularly-polarized light $|R\rangle\left(S_{z}=-1\right)$ couples the excess electron-spin state $|\downarrow\rangle$ to create $X^{-}$in the state $|\downarrow \uparrow \downarrow\rangle$. Here $|\Uparrow\rangle$ and $|\Downarrow\rangle$ represent the heavy-hole spin states $\left|+\frac{3}{2}\right\rangle$ and $\left|-\frac{3}{2}\right\rangle$, respectively.

The input-output relation of this double-sided QD-cavity system can be calculated from the Heisenberg equations of motion for the cavity field operator $\hat{a}$ and $X^{-}$dipole operator $\hat{\sigma}_{-}$in the interaction picture $^{39}$,

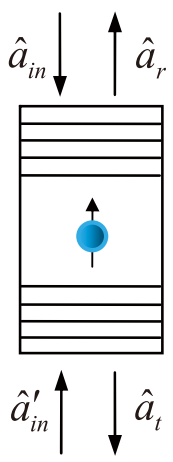

(a)

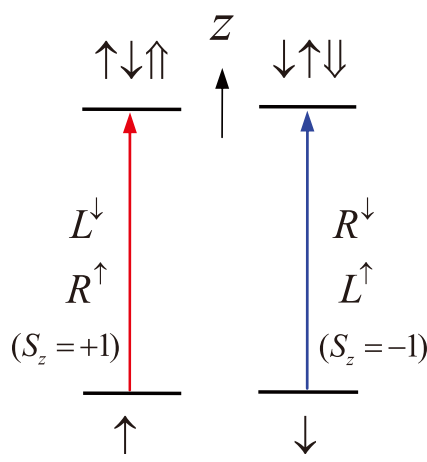

(b)
Figure 1 | (a) A schematic diagram for a singly charged QD inside a double-sided micropillar microcavity interacting with circularly polarized lights. (b) The $X^{-}$spin-dependent optical transition rules due to the Pauli's exclusion principle. $L^{\uparrow}\left(L^{\downarrow}\right)$ and $R^{\uparrow}\left(R^{\downarrow}\right)$ represent the left and the right circularly polarized lights whose input directions are parallel (antiparallel) with $\mathrm{z}$ direction, respectively. $\uparrow$ and $\downarrow$ represent the spins $+\frac{1}{2}$ and $-\frac{1}{2}$ of the excess electron in QD, respectively. $\uparrow \downarrow \uparrow$ and $\downarrow \uparrow \downarrow$ represent the spin states of the negatively charged exciton $X^{-}$.

$$
\begin{aligned}
\frac{d \hat{a}}{d t} & =-\left[i\left(\omega_{c}-\omega\right)+\kappa+\frac{\kappa_{s}}{2}\right] \hat{a}-g \hat{\sigma}_{-}-\sqrt{\kappa} \hat{a}_{i n}-\sqrt{\kappa} \hat{a}_{i n}^{\prime}, \\
\frac{d \hat{\sigma}_{-}}{d t} & =-\left[i\left(\omega_{X^{-}}-\omega\right)+\frac{\gamma}{2}\right] \hat{\sigma}_{-}-g \hat{\sigma}_{z} \hat{a}, \\
\hat{a}_{r} & =\hat{a}_{i n}+\sqrt{\kappa} \hat{a}, \\
\hat{a}_{t} & =\hat{a}_{i n}^{\prime}+\sqrt{\kappa} \hat{a},
\end{aligned}
$$

where $\omega_{X^{-}}, \omega$, and $\omega_{c}$ are the frequencies of the $X^{-}$transition, the input photon, and the cavity mode, respectively. $g$ is the $X^{-}$-cavity coupling strength. $\gamma / 2, \kappa$, and $\kappa_{s} / 2$ are the decay rates of $X^{-}$, the cavity field mode, and the cavity side leakage mode, respectively. $\hat{a}_{i n}, \hat{a}_{i n}^{\prime}$, and $\hat{a}_{r}, \hat{a}_{t}$ are the input and the output field operators, respectively.

In the approximation of a weak excitation condition with $X^{-}$ staying in the ground state at most time and $\left\langle\sigma_{z}\right\rangle=-1$, the reflection and the transmission coefficients of the $X^{-}$-cavity system can be expressed $\mathrm{as}^{25}$,

$$
\begin{aligned}
r(\omega) & =1+t(\omega), \\
t(\omega) & =\frac{-\kappa\left[i\left(\omega_{X^{-}}-\omega\right)+\frac{\gamma}{2}\right]}{\left[i\left(\omega_{X^{-}}-\omega\right)+\frac{\gamma}{2}\right]\left[i\left(\omega_{c}-\omega\right)+\kappa+\frac{\kappa_{s}}{2}\right]+g^{2}} .
\end{aligned}
$$

Considering the resonant condition $\omega_{c}=\omega_{X^{-}}=\omega_{0}$ and the coupling strength $g=0$, the reflection and the transmission coefficients $r_{0}(\omega)$ and $t_{0}(\omega)$ of a cold cavity (the QD is uncoupled to the cavity) are obtained as $^{25}$

$$
\begin{aligned}
& r_{0}(\omega)=\frac{i\left(\omega_{0}-\omega\right)+\frac{\kappa_{s}}{2}}{i\left(\omega_{0}-\omega\right)+\kappa+\frac{\kappa_{s}}{2}}, \\
& t_{0}(\omega)=\frac{-\kappa}{i\left(\omega_{0}-\omega\right)+\kappa+\frac{\kappa_{s}}{2}} .
\end{aligned}
$$

For the input left-circularly-polarized light $|L\rangle\left(S_{z}=+1\right)$, the QD with the electron spin state $|\uparrow\rangle$ is coupled to the cavity (a hot cavity) with the reflection coefficient $|r(\omega)|$ and the transmission coefficient $|t(\omega)|$, whereas the QD with the electron spin state $|\downarrow\rangle$ is uncoupled to the cavity (a cold cavity) with the reflection coefficient $\left|r_{0}(\omega)\right|$ and 
the transmission coefficient $\left|t_{0}(\omega)\right|$. Conversely, for the input rightcircularly-polarized light $|R\rangle\left(S_{z}=-1\right)$, the QD with the electron spin state $|\downarrow\rangle$ is coupled to the cavity, whereas the QD with the electron spin state $|\uparrow\rangle$ is uncoupled to the cavity. In the strong coupling regime $(g>(\kappa, \gamma))$ with the resonant condition, if we adjust the frequencies as $\omega \approx \omega_{0}$ and neglect the cavity side leakage, we get $|r(\omega)| \rightarrow 1,\left|r_{0}(\omega)\right| \rightarrow 0$ and $|t(\omega)| \rightarrow 0,\left|t_{0}(\omega)\right| \rightarrow 1$. That is, the reflection and the transmission operators can be written $\mathrm{as}^{25}$

$$
\begin{aligned}
& \hat{r}(\omega) \simeq r(\omega)(|R\rangle\langle R|| \downarrow\rangle\langle\downarrow|+| L\rangle\langle L|| \uparrow\rangle\langle\uparrow|), \\
& \hat{t}(\omega) \simeq t_{0}(\omega)(|R\rangle\langle R|| \uparrow\rangle\langle\uparrow|+| L\rangle\langle L|| \downarrow\rangle\langle\downarrow|) .
\end{aligned}
$$

Photonic circular polarization is usually dependent on the direction of propagation, so the photon in the state $\left|R^{\dagger}\right\rangle$ or $\left|L^{\downarrow}\right\rangle$ has the $\operatorname{spin} S_{z}=$ +1 , as shown in Fig. 1(b). For the electron spin state $|\uparrow\rangle$, there is the dipole interaction between the electron spin and the photon with the photon state reflected to be $\left|L^{\downarrow}\right\rangle$ or $\left|R^{\dagger}\right\rangle$ by the cavity. For the electron spin state $|\downarrow\rangle$, the input photon is transmitted through cavity with a phase shift relative to the reflected photon, that is ${ }^{27}$,

$$
\begin{array}{ll}
\left|R^{\uparrow}, i_{2}, \uparrow\right\rangle \rightarrow\left|L^{\downarrow}, i_{2}, \uparrow\right\rangle, & \left|L^{\downarrow}, i_{1}, \uparrow\right\rangle \rightarrow\left|R^{\uparrow}, i_{1}, \uparrow\right\rangle, \\
\left|R^{\uparrow}, i_{2}, \downarrow\right\rangle \rightarrow-\left|R^{\uparrow}, i_{1} \downarrow\right\rangle, & \left|L^{\downarrow}, i_{1}, \downarrow\right\rangle \rightarrow-\left|L^{\downarrow}, i_{2}, \downarrow\right\rangle .
\end{array}
$$

In the same way, the reflection and the transmission rules of the photon spin states $\left|R^{\downarrow}\right\rangle$ and $\left|L^{\uparrow}\right\rangle\left(S_{z}=-1\right)$ can be obtained as

$$
\begin{array}{ll}
\left|R^{\downarrow}, i_{1}, \uparrow\right\rangle \rightarrow-\left|R^{\downarrow}, i_{2}, \uparrow\right\rangle, & \left|L^{\uparrow}, i_{2}, \uparrow\right\rangle \rightarrow-\left|L^{\uparrow}, i_{1}, \uparrow\right\rangle, \\
\left|R^{\downarrow}, i_{1}, \downarrow\right\rangle \rightarrow\left|L^{\uparrow}, i_{1}, \downarrow\right\rangle, & \left|L^{\uparrow}, i_{2}, \downarrow\right\rangle \rightarrow\left|R^{\downarrow}, i_{2}, \downarrow\right\rangle .
\end{array}
$$

Here $i_{1}$ and $i_{2}(i=a, b)$ are the two spatial modes of the photon $i$ (shown in Fig. 2).

Spatial-CNOT gate on a two-photon system. The principle of our spatial-CNOT gate is shown in Fig. 2. It is constructed with the reflection and the transmission rules of circularly polarized lights interacting with a QD-cavity system. Suppose that the initial states of the electron spin in QD and the photons $a$ and $b$ are $\frac{1}{\sqrt{2}}(|\uparrow\rangle+|\downarrow\rangle)_{e}, \quad\left(\alpha_{1}|R\rangle+\alpha_{2}|L\rangle\right)_{a}\left(\gamma_{1}\left|a_{1}\right\rangle+\gamma_{2}\left|a_{2}\right\rangle\right)$, and $\left(\beta_{1}|R\rangle+\right.$ $\left.\beta_{2}|L\rangle\right)_{b}\left(\delta_{1}\left|b_{1}\right\rangle+\delta_{2}\left|b_{2}\right\rangle\right)$, respectively.

Before the photon $a$ is put into the quantum circuit shown in Fig. 2, a Hadamard operation is performed on its spatial-mode with a 50:50 beam splitter (BS), which changes the state of the photon $a$ to be $\left|\phi_{a}\right\rangle_{0}=\left(\alpha_{1}|R\rangle+\alpha_{2}|L\rangle\right)_{a}\left(\gamma_{1}^{\prime}\left|a_{1}\right\rangle+\gamma_{2}^{\prime}\left|a_{2}\right\rangle\right)$. Here $\gamma_{1}^{\prime}=\frac{1}{\sqrt{2}}\left(\gamma_{1}+\gamma_{2}\right)$ and $\gamma_{2}^{\prime}=\frac{1}{\sqrt{2}}\left(\gamma_{1}-\gamma_{2}\right)$. The state of the system composed of the

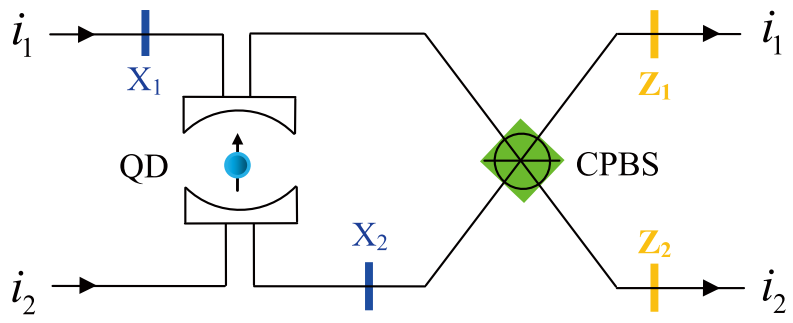

Figure 2 Schematic diagram for a spatial-CNOT gate operating on the spatial-mode DOF of a two-photon system. $X_{i}(i=1,2)$ represents a halfwave plate which is used to perform a polarization bit-flip operation $X=|R\rangle\langle L|+| L\rangle\langle R|$ on the photon passing through it. CPBS represents a polarizing beam splitter in the circular basis, which transmits the photon in the polarization $|R\rangle$ and reflects the photon in the polarization $|L\rangle$, respectively. $Z_{i}(i=1,2)$ represents a half-wave plate which is used to perform a polarization phase-flip operation $Z=-|R\rangle\langle R|+| L\rangle\langle L|$ on a photon.
QD and the photon $a$ is changed from $\left|\phi_{a e}\right\rangle_{0}$ to be $\left|\phi_{a e}\right\rangle_{1}$ after the photon $a$ passes through $\mathrm{X}_{1}, \mathrm{QD}, \mathrm{X}_{2}, \mathrm{CPBS}, \mathrm{Z}_{1}$ and $\mathrm{Z}_{2}$.

$$
\begin{aligned}
& \left|\phi_{a e}\right\rangle_{0}=\frac{1}{\sqrt{2}}(|\uparrow\rangle+|\downarrow\rangle)_{e}\left(\alpha_{1}|R\rangle+\alpha_{2}|L\rangle\right)_{a}\left(\gamma_{1}^{\prime}\left|a_{1}\right\rangle+\gamma_{2}^{\prime}\left|a_{2}\right\rangle\right), \\
& \left|\phi_{a e}\right\rangle_{1}=\frac{-1}{\sqrt{2}}\left[|\uparrow\rangle_{e}\left(\gamma_{2}^{\prime}\left|a_{1}\right\rangle+\gamma_{1}^{\prime}\left|a_{2}\right\rangle\right)-|\downarrow\rangle_{e}\left(\gamma_{1}^{\prime}\left|a_{1}\right\rangle+\gamma_{2}^{\prime}\left|a_{2}\right\rangle\right)\right]\left(\alpha_{1}|R\rangle+\alpha_{2}|L\rangle\right)_{a} .
\end{aligned}
$$

It is not difficult to find that the outcome shown in Eq.(7) is the result of the two-qubit CNOT gate using the electron spin $e$ and the spatialmode of the photon $a$ as the control qubit and the target qubit, respectively.

We perform a Hadamard operation on the electron spin $e$, and put the photon $b$ into the quantum circuit shown in Fig. 2 with its initial state $\left|\phi_{b}\right\rangle_{0}=\left(\beta_{1}|R\rangle+\beta_{2}|L\rangle\right)_{b}\left(\delta_{1}\left|b_{1}\right\rangle+\delta_{2}\left|b_{2}\right\rangle\right)$. The state of the complete system composed of the QD, the photon $a$, and the photon $b$ is changed from $\left|\varphi_{a b e}\right\rangle_{1}$ to be $\left|\varphi_{a b e}\right\rangle_{2}$ after the photon $b$ passes through $Z_{1}$ and $Z_{2}$. Here the same operations are performed on the two photons $a$ and $b$ by the QD-cavity system, $\mathrm{X}_{1}, \mathrm{X}_{2}, \mathrm{CPBS}, \mathrm{Z}_{1}$ and $\mathrm{Z}_{2}$, and the states $\left|\varphi_{a b e}\right\rangle_{1}$ and $\left|\varphi_{a b e}\right\rangle_{2}$ are described as

$$
\begin{aligned}
\left|\varphi_{a b e}\right\rangle_{1}= & \left|\phi_{a e}\right\rangle_{1}\left(\beta_{1}|R\rangle+\beta_{2}|L\rangle\right)_{b}\left(\delta_{1}\left|b_{1}\right\rangle+\delta_{2}\left|b_{2}\right\rangle\right), \\
\left|\varphi_{a b e}\right\rangle_{2}= & \frac{1}{2}\left[-|\uparrow\rangle_{e}\left(\gamma_{1}^{\prime}-\gamma_{2}^{\prime}\right)\left(\left|a_{1}\right\rangle-\left|a_{2}\right\rangle\right)\left(\delta_{1}\left|b_{2}\right\rangle+\delta_{2}\left|b_{1}\right\rangle\right)\right. \\
& \left.+|\downarrow\rangle_{e}\left(\gamma_{1}^{\prime}+\gamma_{2}^{\prime}\right)\left(\left|a_{1}\right\rangle+\left|a_{2}\right\rangle\right)\left(\delta_{1}\left|b_{1}\right\rangle+\delta_{2}\left|b_{2}\right\rangle\right)\right] \\
& \left(\alpha_{1}|R\rangle+\alpha_{2}|L\rangle\right)_{a}\left(\beta_{1}|R\rangle+\beta_{2}|L\rangle\right)_{b} .
\end{aligned}
$$

At last, we perform Hadamard operations on the spatial-mode of the photon $a$ and the electron spin $e$ in sequence, and the state of the complete system becomes

$$
\begin{aligned}
\left|\varphi_{a b e}\right\rangle_{3}= & \frac{1}{\sqrt{2}}\left\{|\uparrow\rangle_{e}\left[\gamma_{1}\left|a_{1}\right\rangle\left(\delta_{1}\left|b_{1}\right\rangle+\delta_{2}\left|b_{2}\right\rangle\right)-\gamma_{2}\left|a_{2}\right\rangle\left(\delta_{1}\left|b_{2}\right\rangle+\delta_{2}\left|b_{1}\right\rangle\right)\right]-\right. \\
& \left.|\downarrow\rangle_{e}\left[\gamma_{1}\left|a_{1}\right\rangle\left(\delta_{1}\left|b_{1}\right\rangle+\delta_{2}\left|b_{2}\right\rangle\right)+\gamma_{2}\left|a_{2}\right\rangle\left(\delta_{1}\left|b_{2}\right\rangle+\delta_{2}\left|b_{1}\right\rangle\right)\right]\right\}^{(9)} \\
& \left(\alpha_{1}|R\rangle+\alpha_{2}|L\rangle\right)_{a}\left(\beta_{1}|R\rangle+\beta_{2}|L\rangle\right)_{b} .
\end{aligned}
$$

The result of spatial-CNOT gate can be achieved by measuring the state of the excess electron spin in the orthogonal basis $\{|\uparrow\rangle,|\downarrow\rangle\}$ and making a feed-forward operation. In detail, if the excess electron spin is in the state $|\uparrow\rangle_{e}$, an additional sign change $\left|a_{2}\right\rangle \rightarrow-\left|a_{2}\right\rangle$ is performed, and the result of spatial-CNOT gate is obtained as

$$
\begin{aligned}
\left|\psi_{a b}\right\rangle= & \frac{1}{2}\left[\gamma_{1}\left|a_{1}\right\rangle\left(\delta_{1}\left|b_{1}\right\rangle+\delta_{2}\left|b_{2}\right\rangle\right)+\gamma_{2}\left|a_{2}\right\rangle\left(\delta_{1}\left|b_{2}\right\rangle+\delta_{2}\left|b_{1}\right\rangle\right)\right] \\
& \left(\alpha_{1}|R\rangle+\alpha_{2}|L\rangle\right)_{a}\left(\beta_{1}|R\rangle+\beta_{2}|L\rangle\right)_{b} .
\end{aligned}
$$

Spatial-polarization hyper-CNOT gate on a two-photon system. We are going to discuss the construction of a spatial-polarization hyper-CNOT gate which performs CNOT gate operations on both the spatial-mode and the polarization DOFs of a two-photon system without using the auxiliary spatial modes or polarization modes.

We have two QD-cavity systems to operate on the two DOFs of the two-photon system $a b$, as shown in Fig. 3. Suppose the two electron spins $e_{1}$ and $e_{2}$ in the two QD-cavity systems (i.e., labeled as $\mathrm{QD}_{1}$ and $\mathrm{QD}_{2}$ in Fig. 3) are prepared in the states $\frac{1}{\sqrt{2}}(|\uparrow\rangle+|\downarrow\rangle)_{e_{1}}$ and $\frac{1}{\sqrt{2}}(|\uparrow\rangle+|\downarrow\rangle)_{e_{2}}$, respectively. The two photons $a$ and $b$ are prepared in the states $\left|\psi_{a}\right\rangle_{0}=\left(\alpha_{1}|R\rangle+\alpha_{2}|L\rangle\right)_{a}\left(\gamma_{1}\left|a_{1}\right\rangle+\gamma_{2}\left|a_{2}\right\rangle\right)$ and $\left|\psi_{b}\right\rangle_{0}=$ $\left(\beta_{1}|R\rangle+\beta_{2}|L\rangle\right)_{b}\left(\delta_{1}\left|b_{1}\right\rangle+\delta_{2}\left|b_{2}\right\rangle\right)$, respectively. We first perform Hadamard operations on both the spatial-mode (with a 50:50 BS, not shown in Fig. 3) and the polarization (with two half-wave plates, not shown in Fig. 3) DOFs of the photon $a$ before we put it into the quantum circuit shown in Fig. 3, and the state of photon $a$ is changed to be $\left|\psi_{a}^{\prime}\right\rangle_{0}=\left(\alpha_{1}^{\prime}|R\rangle+\alpha_{2}^{\prime}|L\rangle\right)_{a}\left(\gamma_{1}^{\prime}\left|a_{1}\right\rangle+\gamma_{2}^{\prime}\left|a_{2}\right\rangle\right)$. Here 


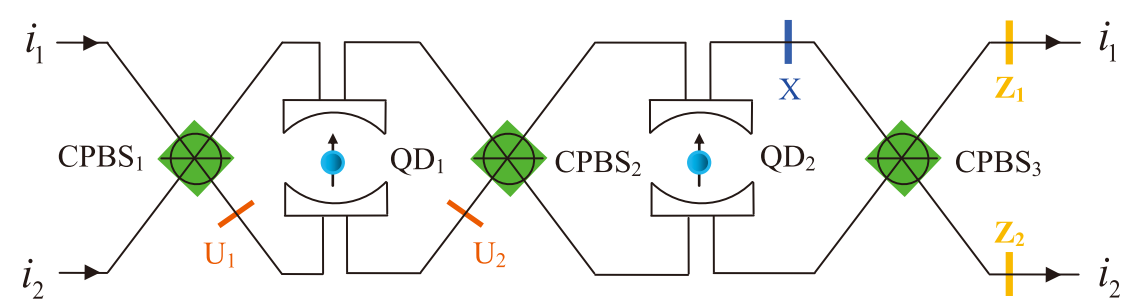

Figure 3 Schematic diagram for a spatial-polarization hyper-CNOT gate operating on both the spatial-mode and the polarization DOFs of a two-photon system. $i_{1}$ and $i_{2}$ represent the two spatial modes of photon $a$ or $b . \mathrm{U}_{i}(i=1,2)$ represents a wave plate which is used to perform a polarization phase-flip operation $U=-|R\rangle\langle R|-| L\rangle\langle L|$ on a photon.

$\alpha_{1}^{\prime}=\frac{1}{\sqrt{2}}\left(\alpha_{1}+\alpha_{2}\right), \quad \alpha_{2}^{\prime}=\frac{1}{\sqrt{2}}\left(\alpha_{1}-\alpha_{2}\right), \quad \gamma_{1}^{\prime}=\frac{1}{\sqrt{2}}\left(\gamma_{1}+\gamma_{2}\right), \quad$ and $\gamma_{2}^{\prime}=\frac{1}{\sqrt{2}}\left(\gamma_{1}-\gamma_{2}\right)$. Subsequently, we let the photon $a$ pass through $\mathrm{CPBS}_{1}, \mathrm{U}_{1}, \mathrm{QD}_{1}, \mathrm{U}_{2}$, and $\mathrm{CPBS}_{2}$ in sequence, and the state of the system composed of $\mathrm{QD}_{1}$ and the photon $a$ is changed from $\left|\Psi_{a e_{1}}\right\rangle_{0}$ to be $\left|\Psi_{a e_{1}}\right\rangle_{1}$. Here

$$
\begin{aligned}
\left|\Psi_{a e_{1}}\right\rangle_{0}= & \frac{1}{\sqrt{2}}(|\uparrow\rangle+|\downarrow\rangle)_{e_{1}}\left(\alpha_{1}^{\prime}|R\rangle+\alpha_{2}^{\prime}|L\rangle\right)_{a}\left(\gamma_{1}^{\prime}\left|a_{1}\right\rangle+\gamma_{2}^{\prime}\left|a_{2}\right\rangle\right), \\
\left|\Psi_{a e_{1}}\right\rangle_{1}= & \frac{1}{\sqrt{2}}\left\{\gamma_{1}^{\prime}\left[|\uparrow\rangle_{e_{1}}\left(\alpha_{1}^{\prime}|L\rangle+\alpha_{2}^{\prime}|R\rangle\right)_{a}+|\downarrow\rangle_{e_{1}}\left(\alpha_{1}^{\prime}|R\rangle+\alpha_{2}^{\prime}|L\rangle\right)_{a}\right]\left|a_{2}\right\rangle\right. \\
& \left.\left.+\gamma_{2}^{\prime}\left[|\uparrow\rangle_{e_{1}}\left(\alpha_{1}^{\prime}|R\rangle+\alpha_{2}^{\prime}|L\rangle\right)_{a}+|\downarrow\rangle_{e_{1}}\left(\alpha_{1}^{\prime}|L\rangle+\alpha_{2}^{\prime}|R\rangle\right)_{a}\right)\right]\left|a_{1}\right\rangle\right\} .
\end{aligned}
$$

We let the photon $a$ pass through $\mathrm{QD}_{2}, \mathrm{X}, \mathrm{CPBS}_{3}, \mathrm{Z}_{1}$, and $\mathrm{Z}_{2}$ in sequence, and the state of the system composed of the photon $a$ and the two electron spins in $\mathrm{QD}_{1}$ and $\mathrm{QD}_{2}$ is changed from $\left|\Phi_{a e_{1} e_{2}}\right\rangle_{1}$ to be $\left|\Phi_{a e_{1} e_{2}}\right\rangle_{2}$ Here

$$
\begin{aligned}
\left|\Phi_{a e_{1} e_{2}}\right\rangle_{1}= & \frac{1}{\sqrt{2}}(|\uparrow\rangle+|\downarrow\rangle)_{e_{2}}\left|\Psi_{a e_{1}}\right\rangle_{1}, \\
\left|\Phi_{a e_{1} e_{2}}\right\rangle_{2}= & \left.\frac{1}{2}\left[|\uparrow\rangle_{e_{1}}\left(\alpha_{1}^{\prime}|R\rangle+\alpha_{2}^{\prime}|L\rangle\right)_{a}+|\downarrow\rangle_{e_{1}}\left(\alpha_{2}^{\prime}|R\rangle+\alpha_{1}^{\prime}|L\rangle\right)_{a}\right)\right] \\
& {\left[|\uparrow\rangle_{e_{2}}\left(\gamma_{2}^{\prime}\left|a_{1}\right\rangle+\gamma_{1}^{\prime}\left|a_{2}\right\rangle\right)-|\downarrow\rangle_{e_{2}}\left(\gamma_{1}^{\prime}\left|a_{1}\right\rangle+\gamma_{2}^{\prime}\left|a_{2}\right\rangle\right)\right] . }
\end{aligned}
$$

This is the result of a four-qubit CNOT gate using the electron spins $e_{1}$ and $e_{2}$ as the control qubits and the polarization and the spatialmode DOFs of the photon $a$ as the target qubits, respectively, and the two CNOT gate operations are performed independently without disturbing the state of photon $a$ in the other DOF.

Subsequently, we let the photon $b$ pass through the quantum circuit shown in Fig. 3 after Hadamard operations are performed on the electron spins $e_{1}$ in $\mathrm{QD}_{1}$ and $e_{2}$ in $\mathrm{QD}_{2}$. The state of the system composed of the two photons and the two electron spins is changed from $\left|\Xi_{a b e_{1} e_{2}}\right\rangle_{2}$ to be $\left|\Xi_{a b e_{1} e_{2}}\right\rangle_{3}$ after the photon $b$ passes through $\mathrm{CPBS}_{1}, \mathrm{U}_{1}, \mathrm{QD}_{1}, \mathrm{U}_{2}, \mathrm{CPBS}_{2}, \mathrm{QD}_{2}, \mathrm{X}, \mathrm{CPBS}_{3}, \mathrm{Z}_{1}$, and $\mathrm{Z}_{2}$. Here the two states $\left|\Xi_{a b e_{1} e_{2}}\right\rangle_{2}$ and $\left|\Xi_{a b e_{1} e_{2}}\right\rangle_{3}$ are described as

$$
\begin{aligned}
\left|\Xi_{a b e_{1} e_{2}}\right\rangle_{2}= & \left|\Phi_{a e_{1} e_{2}}\right\rangle_{2}\left(\beta_{1}|R\rangle+\beta_{2}|L\rangle\right)_{b}\left(\delta_{1}\left|b_{1}\right\rangle+\delta_{2}\left|b_{2}\right\rangle\right), \\
\left|\Xi_{a b e_{1} e_{2}}\right\rangle_{3}= & \frac{1}{2}\left[|\uparrow\rangle_{e_{1}} \alpha_{1}(|R\rangle+|L\rangle)_{a}\left(\beta_{1}|R\rangle+\beta_{2}|L\rangle\right)_{b}\right. \\
& \left.+|\downarrow\rangle_{e_{1}} \alpha_{2}(|R\rangle-|L\rangle)_{a}\left(\beta_{2}|R\rangle+\beta_{1}|L\rangle\right)_{b}\right] \\
& {\left[-|\uparrow\rangle_{e_{2}} \gamma_{2}\left(\left|a_{1}\right\rangle-\left|a_{2}\right\rangle\right)\left(\delta_{2}\left|b_{1}\right\rangle+\delta_{1}\left|b_{2}\right\rangle\right)\right.} \\
& \left.+|\downarrow\rangle_{e_{2}} \gamma_{1}\left(\left|a_{1}\right\rangle+\left|a_{2}\right\rangle\right)\left(\delta_{1}\left|b_{1}\right\rangle+\delta_{2}\left|b_{2}\right\rangle\right)\right] .
\end{aligned}
$$

At last, we perform Hadamard operations on the spatial-mode and the polarization DOFs of the photon $a$ and the excess electron spins in $\mathrm{QD}_{1}$ and $\mathrm{QD}_{2}$ again, and the state of the photon-electron spin system is changed to be

$$
\begin{aligned}
\left|\Xi_{a b e_{1} e_{2}}\right\rangle_{4}= & \frac{1}{2}\left\{|\uparrow\rangle_{e_{1}}\left[\alpha_{1}|R\rangle_{a}\left(\beta_{1}|R\rangle+\beta_{2}|L\rangle\right)_{b}+\alpha_{2}|L\rangle_{a}\left(\beta_{2}|R\rangle+\beta_{1}|L\rangle\right)_{b}\right]\right. \\
& \left.+|\downarrow\rangle_{e_{1}}\left[\alpha_{1}|R\rangle_{a}\left(\beta_{1}|R\rangle+\beta_{2}|L\rangle\right)_{b}-\alpha_{2}|L\rangle_{a}\left(\beta_{2}|R\rangle+\beta_{1}|L\rangle\right)_{b}\right]\right\} \\
& \left\{|\uparrow\rangle_{e_{2}}\left[\gamma_{1}\left|a_{1}\right\rangle\left(\delta_{1}\left|b_{1}\right\rangle+\delta_{2}\left|b_{2}\right\rangle\right)-\gamma_{2}\left|a_{2}\right\rangle\left(\delta_{2}\left|b_{1}\right\rangle+\delta_{1}\left|b_{2}\right\rangle\right)\right]\right. \\
& \left.-|\downarrow\rangle_{e_{2}}\left[\gamma_{1}\left|a_{1}\right\rangle\left(\delta_{1}\left|b_{1}\right\rangle+\delta_{2}\left|b_{2}\right\rangle\right)+\gamma_{2}\left|a_{2}\right\rangle\left(\delta_{2}\left|b_{1}\right\rangle+\delta_{1}\left|b_{2}\right\rangle\right)\right]\right\} .
\end{aligned}
$$

By measuring the two excess electron spins in the orthogonal basis $\{|\uparrow\rangle$, $|\downarrow\rangle\}$ and performing an additional sign change $|L\rangle_{a} \rightarrow-|L\rangle_{a}$ when the electron spin $e_{1}$ is in the state $|\downarrow\rangle_{e_{1}}$ and an additional sign change $\left|a_{2}\right\rangle \rightarrow$ $-\left|a_{2}\right\rangle$ when the electron spin $e_{2}$ is in the state $|\uparrow\rangle_{e_{2}}$, the state of the two-photon system $a b$ becomes

$$
\begin{aligned}
\left|\psi_{a b}\right\rangle= & {\left[\alpha_{1}|R\rangle_{a}\left(\beta_{1}|R\rangle+\beta_{2}|L\rangle\right)_{b}+\alpha_{2}|L\rangle_{a}\left(\beta_{2}|R\rangle+\beta_{1}|L\rangle\right)_{b}\right] } \\
& {\left[\gamma_{1}\left|a_{1}\right\rangle\left(\delta_{1}\left|b_{1}\right\rangle+\delta_{2}\left|b_{2}\right\rangle\right)+\gamma_{2}\left|a_{2}\right\rangle\left(\delta_{2}\left|b_{1}\right\rangle+\delta_{1}\left|b_{2}\right\rangle\right)\right] . }
\end{aligned}
$$

It is not difficult to find that there is a bit flip on the spatial-mode of the photon $b$ (the target qubit) when the spatial-mode of the photon $a$ (the control qubit) is $\left|a_{2}\right\rangle$, compared with the initial state of the twophoton system $a b$. Moreover, a bit flip takes place on the polarization of the photon $b$ when the polarization of the photon $a$ is $|L\rangle$. That is, the quantum circuit shown in Fig. 3 can be used to achieve a hyperCNOT gate operating on the two-photon system $a b$ on both its polarization and its spatial-mode DOFs simultaneously, without auxiliary spatial modes or polarization modes. Moreover, the success probability of this hyper-CNOT gate is $100 \%$ in principle.

\section{Discussion}

In experiment, the transmission and the reflection rules in Eqs.(5) and (6) may fail because of decoherence and dephasing. In this time, the fidelity and the efficiency of our hyper-CNOT gate are reduced. The spin-dependent transition rule is not perfect if we consider the heavy-light hole mixing which can reduce the fidelity by a few percent, while this hole mixing can be reduced by improving the shape, size, and type of QDs ${ }^{26}$. The fidelity can be also reduced in only a few percent by the exciton dephasing, including optical dephasing and hole spin dephasing. The optical coherence time of exciton $(\sim 100 \mathrm{ps})$ is ten times longer than the cavity photon lifetime $\mathrm{e}^{40,41}$, and the hole spin coherence time is three times order of magnitude longer than the cavity photon lifetime $e^{42,43}$. The fine structure splitting, which occurs for neutral exciton, is immune for charged exciton due to the quenched exchange interaction ${ }^{44,45}$. The electron spin decoherence is another factor which can reduce the fidelity, but it can be reduced by extending electron coherence time to $\mu$ s using spin echo techniques, which is longer than the cavity photon lifetime $(\sim 10 p s)^{26}$. The preparation of spin superposition states $|+\rangle$ and $|-\rangle$ can be implemented by using nanosecond electron spin resonance microwave pulses or picosecond optical pulses ${ }^{46}$, of which the preparation time $(p s)$ is significantly shorter than spin coherence time $^{24,47}$. Then the Hadamard operation for transforming electron spin states $|\uparrow\rangle$ and $|\downarrow\rangle$ to $|+\rangle$ and $|-\rangle$ can be achieved. 


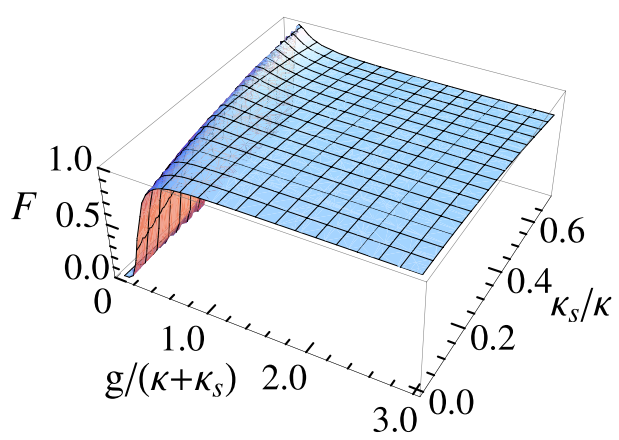

(a)

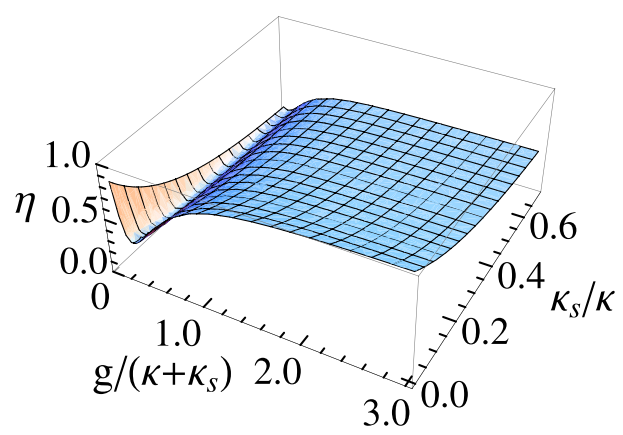

(b)

Figure $4 \mid$ Fidelity and efficiency of the present spatial-polarization hyper-CNOT gate vs the coupling strength and side leakage rate with $\gamma=0.1 \kappa$.

In the resonant condition with $\omega_{c}=\omega_{X^{-}}=\omega_{0}=\omega$, if the cavity side leak is taken into account, the optical selection rules for a QDcavity system given by Eqs. (5) and (6) become ${ }^{25}$

$$
\begin{aligned}
& \left|R^{\uparrow}, i_{2}, \uparrow\right\rangle \rightarrow|r|\left|L^{\downarrow}, i_{2}, \uparrow\right\rangle-|t|\left|R^{\uparrow}, i_{1}, \uparrow\right\rangle, \\
& \left|L^{\downarrow}, i_{1}, \uparrow\right\rangle \rightarrow|r|\left|R^{\uparrow}, i_{1}, \uparrow\right\rangle-|t|\left|L^{\downarrow}, i_{2}, \uparrow\right\rangle, \\
& \left|R^{\downarrow}, i_{1}, \downarrow\right\rangle \rightarrow|r|\left|L^{\uparrow}, i_{1}, \downarrow\right\rangle-|t|\left|R^{\downarrow}, i_{2}, \downarrow\right\rangle, \\
& \left|L^{\uparrow}, i_{2}, \downarrow\right\rangle \rightarrow|r|\left|R^{\downarrow}, i_{2}, \downarrow\right\rangle-|t|\left|L^{\uparrow}, i_{1}, \downarrow\right\rangle, \\
& \left|R^{\downarrow}, i_{1}, \uparrow\right\rangle \rightarrow-\left|t_{0}\right|\left|R^{\downarrow}, i_{2}, \uparrow\right\rangle+\left|r_{0}\right|\left|L^{\uparrow}, i_{1}, \uparrow\right\rangle, \\
& \left|L^{\uparrow}, i_{2}, \uparrow\right\rangle \rightarrow-\left|t_{0}\right|\left|L^{\uparrow}, i_{1}, \uparrow\right\rangle+\left|r_{0}\right|\left|R^{\downarrow}, i_{2}, \uparrow\right\rangle, \\
& \left|R^{\uparrow}, i_{2}, \downarrow\right\rangle \rightarrow-\left|t_{0}\right|\left|R^{\uparrow}, i_{1}, \downarrow\right\rangle+\left|r_{0}\right|\left|L^{\downarrow}, i_{2}, \downarrow\right\rangle, \\
& \left|L^{\downarrow}, i_{1}, \downarrow\right\rangle \rightarrow \quad-\left|t_{0}\right|\left|L^{\downarrow}, i_{2}, \downarrow\right\rangle+\left|r_{0}\right| \mid R^{\left.\uparrow, i_{1}, \downarrow\right\rangle .}
\end{aligned}
$$

The fidelity of a quantum logical gate is defined as $F=\left|\left\langle\psi_{f} \mid \psi\right\rangle\right|^{2}$, where $|\psi\rangle$ is the ideal final state of the quantum system after the process for a quantum logical gate, and $\left|\psi_{f}\right\rangle$ is the final state of the system by considering external reservoirs. The efficiency of a photonic logical gate is defined as the probability of the photons to be detected after the logic gate operation. The fidelity and the efficiency of the present spatial-polarization hyper-CNOT gate are

$$
\begin{aligned}
F & =\frac{\left(\xi_{1} \zeta_{1} m\right)^{2}}{4\left(\left|\xi_{1} \zeta_{2}\right|^{2}+\left|\xi_{1} \xi_{2}\right|^{2}\right) n_{1}+4\left(\left|\xi_{1} \zeta_{1}\right|^{2}+\left|\xi_{2}^{2}\right|^{2}\right) n_{2}} \\
\eta & =\frac{1}{16}\left(|r|^{2}+|t|^{2}+\left|t_{0}\right|^{2}+\left|r_{0}\right|^{2}\right)^{4}
\end{aligned}
$$

Here

$$
\begin{aligned}
\zeta_{1}= & \left|t_{0}\right|+|r|-\left|r_{0}\right|-|t|, \zeta_{2}=|t|-|r|-\left|r_{0}\right|+\left|t_{0}\right|, \\
\xi_{1}= & \left|t_{0}\right|+\left|r_{0}\right|+|r|+|t|, \xi_{2}=|r|+|t|-\left|r_{0}\right|-\left|t_{0}\right|, \\
m= & \left(\left|t_{0}\right|+\left|r_{0}\right|\right)\left|t_{0}\right|-(|r|+|t|)\left|r_{0}\right|+\left(\left|t_{0}\right|+\left|r_{0}\right|\right)|r| \\
& -(|r|+|t|)|t|-\left(\left|t_{0}\right|+\left|r_{0}\right|\right)\left|r_{0}\right|+(|r|+|t|)\left|t_{0}\right| \\
& -\left(\left|t_{0}\right|+\left|r_{0}\right|\right)|t|+(|r|+|t|)|r|, \\
n_{1}= & \left.|(|t|+|r|)| t_{0}\left|-\left(\left|r_{0}\right|+\left|t_{0}\right|\right)\right| r_{0}\right|^{2}+|(|t|+|r|)| r \mid- \\
& \left(\left|r_{0}\right|+\left|t_{0}\right|\right)|t|^{2}+\left.|(|t|+|r|)| r_{0}\left|-\left(\left|r_{0}\right|+\left|t_{0}\right|\right)\right| t_{0}\right|^{2} \\
& +|(|t|+|r|)| t\left|-\left(\left|r_{0}\right|+\left|t_{0}\right|\right)\right| r \|^{2}, \\
n_{2}= & \left|\left(\left|t_{0}\right|+\left|r_{0}\right|\right)\right| t_{0}|-(|r|+|t|)| r_{0} \|^{2}+\left|\left(\left|t_{0}\right|+\left|r_{0}\right|\right)\right| r \mid \\
& -(|r|+|t|)|t|^{2}+\left|\left(\left|t_{0}\right|+\left|r_{0}\right|\right)\right| r_{0}|-(|r|+|t|)| t_{0} \|^{2} \\
& +\left|\left(\left|t_{0}\right|+\left|r_{0}\right|\right)\right| t|-(|r|+|t|)| r \|^{2} .
\end{aligned}
$$

The fidelity and the efficiency are mainly affected by the coupling strength and the cavity side leakage as shown in Fig. 4. The strong coupling strength and the low side leakage and cavity loss rate $\left(\kappa_{s} / \kappa\right)$ are required for the present spatial-polarization hyperCNOT quantum gate with a high fidelity and a high efficiency. The strong coupling strength $g /\left(\kappa+\kappa_{s}\right) \simeq 0.8$ has been observed in a large micropillar $(d=7.3 \mu \mathrm{m})^{48}$ with a quality factor of $Q \sim$ $6.5 \times 10^{4}$ in a current experiment. In $d=1.5 \mu \mathrm{m}$ micropillar microcavity, the coupling strength $g /\left(\kappa+\kappa_{s}\right) \simeq 0.5$ was reported $^{49}$ with $Q \sim 8800$, and the coupling strength $g /\left(\kappa+\kappa_{s}\right) \simeq 2.4(Q \sim 4$ $\times 10^{4}$ ) could be achieved ${ }^{50}$ by improving the sample designs, growth, and fabrication ${ }^{51}$. In the case $g /\left(\kappa+\kappa_{s}\right) \simeq 0.5$ and $\kappa_{s} / \kappa \simeq 0$, the fidelity and the efficiency for this hyper-CNOT gate are $F=92.5 \%$ and $\eta=55 \%$, respectively. If the coupling strength is $g /\left(\kappa+\kappa_{s}\right) \simeq 2.4$ with $\kappa_{s} / \kappa \simeq 0$, the fidelity and the efficiency become $F=100 \%$ and $\eta=97 \%$, respectively. If the side leakage and cavity loss rate is $\kappa_{s} / \kappa \simeq 0.3$ for $g /\left(\kappa+\kappa_{s}\right) \simeq 2.4$, the fidelity and the efficiency are $F=96 \%$ and $\eta=60.3 \%$. The fidelity and the efficiency of this hyper-CNOT gate are mainly reduced by a weak coupling strength and a high cavity side leakage. The quality factor of a micropillar microcavity is dominated by the side leakage and cavity loss rate $\kappa_{s} / \kappa$. The side leakage and cavity loss rate has been reduced to $\kappa_{s} / \kappa \simeq 0.7$ with the quality factor reduced to $Q \sim 1.7 \times 10^{4}\left(g /\left(\kappa+\kappa_{s}\right) \simeq 1\right)$ in the micropillar $d=1.5 \mu \mathrm{m}$ by thinning down the top mirrors ${ }^{26}$. High efficiency operations are required to achieve a stronger coupling strength with a lower side leakage in micropillars.

In our previous work, we introduced a hyper-CNOT gate constructed with the reflection geometry optical property of one-side QD-cavity systems with one mirror partially reflective and another mirror $100 \%$ reflective ${ }^{52}$. The hyper-CNOT gate in one-side QDcavity protocol is fragile because the reflectance for the uncoupled cavity and the coupled cavity should be balanced to get high fidelity ${ }^{25}$. The present hyper-CNOT gate is constructed with the reflection/ transmission geometry optical property of double-sided QD-cavity systems with both mirrors partially reflective. And this hyper-CNOT gate is robust and flexible with the large reflectance and transmittance difference between the uncoupled cavity and the coupled cavity $^{25}$. Moreover, in the one-side QD-cavity protocol, the problem about how to put the several spatial modes of a photon into the single-sided cavity is required to be solved. However, this is not a problem for this double-sided QD-cavity protocol, because there are two balanced spatial modes for a double-sided cavity. And the operations on the two spatial modes are combined in this double-sided QD-cavity protocol, especially for the Mach-Zehnder interferometer 
induced by the CPBSs. That is, both the fidelity and the efficiency of the present hyper-CNOT gate are higher than the one in one-side QD-cavity protocol.

With the hyper-CNOT gate operation, the quantum information processing can be implemented with less photon resources, which can reduce the resources required in the quantum logic circuits and the storage process. And the photonic dissipation and the environment noise effect are depressed in the quantum information processing based on two DOFs of photon systems. Moreover, the interaction times between the photons is decreased in the quantum information protocols by using the two DOFs of photon systems, compared with the integration of several cascaded CNOT gates in one DOF. For instance, in the preparation of four-qubit cluster states, only a hyper-CNOT gate operation (photons interact with electron spins four times) and a wave plate are required in the protocol with two photons in two $\mathrm{DOFs}^{52,53}$, while three CNOT gate operations (photons interact with electron spins six times) are required in the protocol with four photons in one DOF. In this article, we have only discussed the construction of a spatial-polarization hyper-CNOT gate with the nonlinear optics of double-sided QD-cavity system. With the present hyper-CNOT gate and single-photon quantum gates, a hyper-parallel photonic quantum computation can be constructed in principle, and the preparation and analysis of multiphoton hyperentangled states can also be implemented in a simple way. In principle, some other quantum systems with nonlinear optics can also be used to achieve scalable hyper-parallel quantum computation, such as wave-guide nanocavity, nitrogen-vacancy centers, Rydberg atoms, cross-Kerr media, and so on. Here the hyper-CNOT gate operation is used to operate on the polarization and the spatialmode DOFs independently, and the two photons with two DOFs are used as four qubits. In fact, the two photons with two DOFs can also be used as two qudits in quantum information processing. Moreover, as the CNOT gate on the two DOFs of a photon can be implemented easily with the linear optical elements and the single-qubit gates for the two DOFs of a photon can be implemented independently, the multiqubit hybrid logic gate can be implemented in a simpler way with less photon resources resorting to the two DOFs of the photon system.

In summary, we have constructed the spatial-polarization hyperCNOT gate operating on both the spatial-mode and the polarization DOFs of a two-photon system with the giant optical circular birefringence of quantum dot inside double-sided optical microcavity. This spatial-polarization hyper-CNOT gate may work efficiently in strong coupling regimes with low cavity side leakage. In the construction of the hyper-CNOT gate, the two DOFs of a two-photon system are used to assist each other to implement the CNOT gate operations without using the auxiliary spatial modes, which is much simpler than the one constructed with the quantum dots inside one-side optical microcavities ${ }^{52}$. The measurement on the electron spins in the QDs can be replaced by making the control qubits of the photon system interact with QD-cavity systems twice as that introduced by Duan and Kimble ${ }^{15}$, but this will decrease the fidelity and the efficiency of the hyper-CNOT gate. Therefore, it relies on the experimental technique for deciding whether people use the measurement on electron spins or not.

\section{Methods}

Measurement on electron spin $e$ in QD. The measurement on the electron spin $e$ can be implemented by using an auxiliary photon. If the auxiliary photon is initially in the state $\left|\psi_{i}\right\rangle=\left|R_{i}\right\rangle\left|i_{1}\right\rangle$, after interacting with QD-cavity system, the final state of the complete system becomes

$$
\begin{aligned}
& \left|R_{i}\right\rangle\left|i_{1}\right\rangle|\uparrow\rangle_{e} \rightarrow-\left|R_{i}\right\rangle\left|i_{2}\right\rangle|\uparrow\rangle_{e}, \\
& \left|R_{i}\right\rangle\left|i_{1}\right\rangle|\downarrow\rangle_{e} \rightarrow\left|L_{i}\right\rangle\left|i_{1}\right\rangle|\downarrow\rangle_{e} .
\end{aligned}
$$

If the auxiliary photon is detected on the spatial mode $\left|i_{2}\right\rangle$, the electron spin is projected into the state $|\uparrow\rangle_{e}$, whereas the electron spin is projected into the state $|\downarrow\rangle_{e}$ with the detection of the auxiliary photon on the spatial mode $\left|i_{1}\right\rangle$.
Spin echo technique using single photon pulse. Our schemes, implemented with the optical properties of QD-cavity system, are not compatible with ESR-based spin echo technique in an external magnetic field, which may cause the detuning between the two spin states. In 2011, Hu and Rarity ${ }^{26}$ showed that the microwave pulses or optical pulses used in spin echo techniques may be replaced by the single photon pulses to suppress the nuclear spin fluctuations and hyperfine interaction. That is, the single photons can play the role of the $\pi$-pulse to make the spin rotate $180^{\circ}$ around the optical axis. If a photon in the state $\frac{1}{\sqrt{2}}(|R\rangle+|L\rangle)_{i}\left|i_{1}\right\rangle$ is put in to the CPBS, QD and CPBS in sequence, after interaction, the state of the system composed of the photon $i$ and the electron spin $e$ in QD is changed as

$$
\frac{1}{2}(|R\rangle+|L\rangle)_{i}\left|i_{1}\right\rangle(|\uparrow\rangle+|\downarrow\rangle)_{e} \rightarrow \frac{1}{2}(|R\rangle+|L\rangle)_{i}\left|i_{2}\right\rangle(|\uparrow\rangle-|\downarrow\rangle)_{e} .
$$

Double-sided QD-cavity system in quantum information processing based on two DOFs. As the spatial-mode and the polarization DOFs of a photon system can be converted into each other easily, the logic gate operation on the polarization (spatialmode) DOF may be simplified by assisting with the spatial-mode (polarization) DOF, especially for the interaction between the two photons. There are many works on the polarization logic gates using the spatial-mode DOF as the assistant $t^{13-15,27-30}$. The double-sided QD-cavity system has two spatial modes, which are always used as assistant in manipulating the polarization DOF and at last be consumed ${ }^{27-30}$. The spatial-mode and the polarization DOFs of a photon can be manipulated independently and easily with linear optical elements, so it is convenient to use these two DOFs as two qubits in quantum information processing. Our hyper-CNOT gate shows that the spatial-mode and the polarization DOFs can assist each other to perform CNOT gate operations on the photonic states of these two DOFs independently without auxiliary spatial modes, resorting to the optical property shown in Eqs.(5) and (6). This is simpler than the one using one-side QD-cavity system $^{52}$. Of course, the two DOFs of a photon can be used as a qudit, and the doublesided QD-cavity system can also be used to implement the quantum information processing with multiple qudits in two DOFs. It may be convenient to use doublesided QD-cavity system to implement the quantum information processing with two DOFs.

1. Nielsen, M. A. \& Chuang, I. L. Quantum Computation and Quantum Information (Cambridge University Press, Cambridge, England, 2000).

2. Gisin, N., Ribordy, G., Tittel, W. \& Zbinden, H. Quantum cryptography. Rev. Mod. Phys. 74, 145-195 (2002).

3. Schmidt-Kaler, F. et al. Realization of the Cirac-Zoller controlled-NOT quantum gate. Nature 422, 408-411 (2003).

4. Beenakker, C. W. J., DiVincenzo, D. P., Emary, C. \& Kindermann, M. Charge detection enables free-electron quantum computation. Phys. Rev. Lett. 93, 020501 (2004).

5. Turchette, Q. A., Hood, C. J., Lange, W., Mabuchi, H. \& Kimble, H. J. Measurement of conditional phase shifts for quantum logic. Phys. Rev. Lett. 75 , 4710-4713 (1995).

6. Rauschenbeutel, A. et al. Coherent operation of a tunable quantum phase gate in cavity QED. Phys. Rev. Lett. 83, 5166-5169 (1999).

7. Gershenfeld, N. A. \& Chuang, I. L. Bulk spin-resonance quantum computation. Science 275, 350-356 (1997).

8. Jones, J. A., Mosca, M. \& Hansen, R. H. Implementation of a quantum search algorithm on a quantum computer. Nature 393, 344-346 (1998).

9. Feng, G., Xu, G. \& Long, G. Experimental realization of nonadiabatic Holonomic quantum computation. Phys. Rev. Lett. 110, 190501 (2013).

10. Li, X. et al. An all-optical quantum gate in a semiconductor quantum dot. Science 301, 809-811 (2003).

11. Yamamoto, T., Pashkin, Y. A., Astafiev, O., Nakamura, Y. \& Tsai, J. S. Demonstration of conditional gate operation using superconducting charge qubits. Nature 425, 941-944 (2003).

12. Knill, E., Laflamme, R. \& Milburn, G. J. A scheme for efficient quantum computation with linear optics. Nature 409, 46-52 (2001).

13. O’Brien, J. L., Pryde, G. J., White, A. G., Ralph, T. C. \& Branning, D. Demonstration of an all-optical quantum controlled-NOT gate. Nature 426, 264-267 (2003).

14. Nemoto, K. \& Munro, W. J. Nearly deterministic linear optical controlled-NOT gate. Phys. Rev. Lett. 93, 250502 (2004).

15. Duan, L.-M. \& Kimble, H. J. Scalable photonic quantum computation through cavity-assisted interactions. Phys. Rev. Lett. 92, 127902 (2004).

16. Koshino, K., Ishizaka, S. \& Nakamura, Y. Deterministic photon-photon $\sqrt{S W A P}$ gate using a $\Lambda$ system. Phys. Rev. A 82, 010301(R) (2010).

17. Fleischhauer, M., Imamoglu, A. \& Marangos, J. P. Electromagnetically induced transparency: optics in coherent media. Rev. Mod. Phys. 77, 633-673 (2005).

18. Schmidt, H. \& Imamogdlu, A. Giant Kerr nonlinearities obtained by electromagnetically induced transparency. Opt. Lett. 21, 1936-1938 (1996).

19. Sheng, Y. B., Deng, F. G. \& Long, G. L. Complete hyperentangled-Bell-state analysis for quantum communication. Phys. Rev. A 82, 032318 (2010). 
20. Hofmann, H. F., Kojima, K., Takeuchi, S. \& Sasaki, K. Optimized phase switching using a single-atom nonlinearity. J. Opt. B: Quantum Semiclass. Opt. 5, 218-221 (2003).

21. Menicucci, N. C., Flammia, S. T. \& Pfister, O. One-way quantum computing in the optical frequency comb. Phys. Rev. Lett. 101, 130501 (2008).

22. Langford, N. K. et al. Efficient quantum computing using coherent photon conversion. Nature 478, 360-363 (2011).

23. Hu, C. Y., Young, A., O’Brien, J. L., Munro, W. J. \& Rarity, J. G. Giant optical Faraday rotation induced by a single-electron spin in a quantum dot: applications to entangling remote spins via a single photon. Phys. Rev. B 78, 085307 (2008).

24. Hu, C. Y., Munro, W. J. \& Rarity, J. G. Deterministic photon entangler using a charged quantum dot inside a microcavity. Phys. Rev. B 78, 125318 (2008).

25. Hu, C. Y., Munro, W. J., O’Brien, J. L. \& Rarity, J. G. Proposed entanglement beam splitter using a quantum-dot spin in a double-sided optical microcavity. Phys. Rev. B 80, 205326 (2009).

26. Hu, C. Y. \& Rarity, J. G. Loss-resistant state teleportation and entanglement swapping using a quantum-dot spin in an optical microcavity. Phys. Rev. B 83, 115303 (2011).

27. Bonato, C. et al. CNOT and Bell-state analysis in the weak-coupling cavity QED regime. Phys. Rev. Lett. 104, 160503 (2010).

28. Wei, H. R. \& Deng, F. G. Universal quantum gates for hybrid systems assisted by quantum dots inside double-sided optical microcavities. Phys. Rev. A 87, 022305 (2013).

29. Wei, H. R. \& Deng, F. G. Scalable photonic quantum computing assisted by quantum-dot spin in double-sided optical microcavity. Opt. Express 21, 17671-17685 (2013).

30. Wang, H. F., Zhu, A. D., Zhang, S. \& Yeon, K. H. Deterministic CNOT gate and entanglement swapping for photonic qubits using a quantum-dot spin in a double-sided optical microcavity. arXiv 1309.0410 (2013).

31. Wei, H. R. \& Deng, F. G. Universal quantum gates on electron-spin qubits with quantum dots inside single-side optical microcavities. Opt. Express 22, 593-607 (2014).

32. Ren, B. C., Wei, H. R., Hua, M., Li, T. \& Deng, F. G. Complete hyperentangledBell-state analysis for photon systems assisted by quantum-dot spins in optical microcavities. Opt. Express 20, 24664-24677 (2012).

33. Wang, T. J., Lu, Y. \& Long, G. L. Generation and complete analysis of the hyperentangled Bell state for photons assisted by quantum-dot spins in optical microcavities. Phys. Rev. A 86, 042337 (2012).

34. Wang, T. J., Song, S. Y. \& Long, G. L. Quantum repeater based on spatial entanglement of photons and quantum-dot spins in optical microcavities. Phys. Rev. A 85, 062311 (2012).

35. Sleator, T. \& Weinfurter, H. Realizable universal quantum logic gates. Phys. Rev. Lett. 74, 4087-4090 (1995).

36. Gudat, J. et al. Permanent tuning of quantum dot transitions to degenerate microcavity resonances. Appl. Phys. Lett. 98, 121111 (2011).

37. Warburton, R. J. et al. Charged excitons in self-assembled semiconductor quantum dots. Phys. Rev. Lett. 79, 5282-5285 (1997).

38. Hu, C. Y. et al. Optically detected magnetic resonance of excess electrons in type-I quantum wells with a low-density electron gas. Phys. Rev. B 58, R1766-R1769 (1998).

39. Walls, D. F. \& Milburn, G. J. Quantum Optics (Springer-Verlag, Berlin, 1994).

40. Borri, P. et al. Ultralong dephasing time in InGaAs quantum dots. Phys. Rev. Lett. 87, 157401 (2001).
41. Birkedal, D., Leosson, K. \& Hvam, J. M. Long lived coherence in self-assembled quantum dots. Phys. Rev. Lett. 87, 227401 (2001).

42. Gerardot, B. D. et al. Optical pumping of a single hole spin in a quantum dot. Nature 451, 441-444 (2008).

43. Brunner, D. et al. A coherent single-hole spin in a semiconductor. Science 325, 70-72 (2009).

44. Bayer, M. et al. Fine structure of neutral and charged excitons in self-assembled In(Ga)As/(Al)GaAs quantum dots. Phys. Rev. B 65, 195315 (2002).

45. Finley, J. J. et al. Fine structure of charged and neutral excitons in InAs$\mathrm{Al}_{0.6} \mathrm{Ga}_{0.4}$ As quantum dots. Phys. Rev. B 66, 153316 (2002).

46. Press, D., Ladd, T. D., Zhang, B. \& Yamamoto, Y. Complete quantum control of a single quantum dot spin using ultrafast optical pulses. Nature 456, 218-221 (2008).

47. Kimble, H. J. Cavity Quantum Electrodynamics (Academic, San Diego, 1994).

48. Loo, V. et al. Quantum dot-cavity strong-coupling regime measured through coherent reflection spectroscopy in a very high-Q micropillar. Appl. Phys. Lett. 97, 241110 (2010).

49. Reithmaier, J. P. et al. Strong coupling in a single quantum dot-semiconductor microcavity system. Nature 432, 197-200 (2004).

50. Yoshie, T. et al. Vacuum Rabi splitting with a single quantum dot in a photonic crystal nanocavity. Nature 432, 200-203 (2004)

51. Reitzenstein, S. et al. AlAs/GaAs micropillar cavities with quality factors exceeding 150.000. Appl. Phys. Lett. 90, 251109 (2007).

52. Ren, B. C., Wei, H. R. \& Deng, F. G. Deterministic photonic spatial-polarization hyper-controlled-not gate assisted by a quantum dot inside a one-side optical microcavity. Laser Phys. Lett. 10, 095202 (2013).

53. Ren, B. C., Du, F. F. \& Deng, F. G. Hyperentanglement concentration for twophoton four-qubit systems with linear optics. Phys. Rev. A 88, 012302 (2013).

\section{Acknowledgments}

This work was supported by the National Natural Science Foundation of China under Grant No. 11174039 and NECT-11-0031.

\section{Author contributions}

B.C. and F.G. wrote the main manuscript text and B.C. prepared figures 1-4. Both authors reviewed the manuscript.

\section{Additional information}

Competing financial interests: The authors declare no competing financial interests.

How to cite this article: Ren, B.-C. \& Deng, F.-G. Hyper-parallel photonic quantum computation with coupled quantum dots. Sci. Rep. 4, 4623; DOI:10.1038/srep04623 (2014).

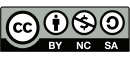

This work is licensed under a Creative Commons Attribution-NonCommercialShareAlike 3.0 Unported License. The images in this article are included in the article's Creative Commons license, unless indicated otherwise in the image credit; if the image is not included under the Creative Commons license, users will need to obtain permission from the license holder in order to reproduce the image. To view a copy of this license, visit http://creativecommons.org/licenses/by-nc-sa/3.0/ 